\title{
Primitive Neuroectodermal Tumor in an Ovarian Cystic Teratoma: Natural Killer and Neuroblastoma Cell Analysis
}

\author{
Giovanna Tabellini $^{\mathrm{a}} \quad$ Marzia Benassi $^{\mathrm{a}}$ Emanuela Marcenaro $^{c}$ \\ Daniela Coltrini $^{\mathrm{a}} \quad$ Ornella Patrizi $^{\mathrm{a}} \quad$ Doris Ricotta $^{\mathrm{a}}$ Fabio Rampinelli ${ }^{\mathrm{b}}$ \\ Alessandro Moretta ${ }^{c}$ Silvia Parolini ${ }^{a}$ \\ a Dipartimento di Medicina Molecolare e Traslazionale, Università degli Studi di Brescia, \\ and ${ }^{b}$ Dipartimento di Ostetricia e Ginecologia, Spedali Civili di Brescia, Brescia, and \\ 'Dipartimento di Medicina Sperimentale, Centro di Eccellenza per le Ricerche Biomediche, \\ Università degli Studi di Genova, Genoa, Italy
}

\section{Key Words}

Activating natural killer receptors · Natural killer cells $\cdot$ Neuroblastoma $\cdot$ Ovarian cancer

\begin{abstract}
In the present study, we report an extremely rare case of a 31-year-old woman with neuroblastoma arising in an ovarian cystic teratoma. We analyzed the expression of activating receptors on natural killer (NK) cells derived from the patient's peripheral blood and peritoneal fluid. In addition, we investigated the presence of specific ligands recognized by different NK cell receptors on tumor cells. We show that NK cells isolated from peritoneal fluid expressed certain triggering receptors including DNAM-1 (CD226) and CD16 with lower intensity as compared to peripheral blood NK cells. Remarkably, at variance with most cases of childhood neuroblastoma, the tumor cells from this patient expressed substantial amounts of HLA class-I molecules. These molecules are known to be protective against NK cellmediated lysis. In addition, neuroblastoma cells expressed B7-H3 (CD276), another surface molecule that inhibits NK cell function. Finally, this tumor did not express the PVR (CD155) and nectin-2 (CD112) ligands for the DNAM-1 activating NK receptor, which plays a crucial role in NK/neuroblastoma interactions. Altogether, these findings indicate that the neuroblastoma cells of this patient express an NK-resistant surface phenotype, which is at least in part similar to that previously described in a fraction of childhood neuroblastoma.
\end{abstract}


Tabellini et al.: Primitive Neuroectodermal Tumor in an Ovarian Cystic Teratoma: Natural Killer and Neuroblastoma Cell Analysis

\section{Introduction}

Primary malignant neuroectodermal tumors of the ovary, microscopically identical to their neoplastic counterparts in the nervous system, are extremely rare and arise from primitive neuroectodermal cells of neural crest with variable degrees of differentiation [13]. Neural tissue occurs with considerable frequency in mature cystic teratoma but the development of a malignant neural neoplasm in an ovarian tumor of this type is an event of exceptional rarity. Only 9 cases with a primary ovarian location have been published in the literature since 1982 [4]. Natural killer (NK) lymphocytes represent one of the most efficient cellular mechanisms by which the immune system can destroy tumor or virally infected cells $[5,6]$. The function of NK cells is controlled by an array of different activating [7] and inhibitory receptors $[6,8]$ that, upon engagement by specific cell ligands on target cells, may either induce or suppress the process of killing. The inhibitory receptors, including inhibitory killer Ig-like receptors (iKIRs) (specific for allotypic determinants of HLA-C, -B and -A molecules) and the HLA-E-specific CD94/NKG2A [8], sense the expression of HLA class-I molecules on potential target cells. The lack of appropriate receptor/HLA interactions may lead to NK-mediated killing of cells with defective HLA class-I expression. NB cells have been shown to represent excellent targets for NK-mediated cytotoxicity, depending on the lack of expression of surface HLA class-I molecules and on the presence of specific ligands recognized by the activating NK cell receptors NCRs (NKp46 and NKp30), NKG2D and DNAM-1 $[6,7,9,10]$. Here, we report a rare case of a 31-year-old woman with neuroblastoma arising in ovarian mature cystic teratoma [11]. In this study, we characterized the surface expression of activating receptors on NK cells isolated from peripheral blood (PB) and peritoneal fluid (PF) of the patient, and we analyzed the expression of relevant ligands for NK receptors on autologous neuroblasts.

\section{Materials and Methods}

\section{Case Presentation}

A 31-year-old, gravida 3, para 3, Caucasian woman, was referred with abdominal distension and general fatigue for 2 months. Pelvic ultrasound and computed tomography of the pelvis and abdomen showed a large left ovarian mass measuring $17 \times 11 \mathrm{~cm}$ in diameter. Her preoperative serum cancer markers showed an elevated cancer antigen 125 (Ca125) level of $102 \mathrm{U} / \mathrm{ml}$ (normal range $<35 \mathrm{U} / \mathrm{ml}$ ) and a lactate dehydrogenase level of $511 \mathrm{U} / \mathrm{l}$ (normal range 125-220 U/l). Serum levels of cancer antigen 19.9, CEA and calcium were within normal ranges. She underwent exploratory laparotomy, which revealed a large, solid left ovarian mass with diffuse and nodular disseminations on the peritoneum, omentum, with retroperitoneal pelvic and periaortic lymph nodes enlargement. The left ovarian mass was removed and sent for pathologic evaluation. Immunohistochemistry showed positive cells for synaptophysin, neuron-specific enolase, chromogranin, CD56 and neurofilaments and confirmed the diagnosis of neuroblastoma arising in ovarian teratoma.

Written informed consent for publication of this case report was obtained from the patient. Approval was obtained from the Review Board of the Spedali Civili of Brescia. Informed consent was provided according to the Declaration of Helsinki.

Monoclonal Antibodies

The following mAbs generated in our laboratories (DIMES, University of Genoa and Department of Molecular and Translational Medicine, University of Brescia), were used in 
Tabellini et al.: Primitive Neuroectodermal Tumor in an Ovarian Cystic Teratoma: Natural Killer and Neuroblastoma Cell Analysis

this study: BAB281 (IgG1 anti-NKp46), AZ20 (IgG1 anti-NKp30), ON72 (IgG1 anti-NKG2D), SUS142 (IgG2b anti-CD16), C227 (IgG1 anti-CD69), FS123 (IgG2a anti-DNAM-1), C218 (IgG1 anti-CD56), 11PB6 (IgG1 anti-KIR2DL1/S1), GL183 (IgG1 anti-KIR2DL2/L3/S2), AZ158 (IgG2a anti-KIR3DL1/S1/L2), Z199 (IgG2b anti-CD94/NKG2A), 5A10 (IgG1 anti-PVR), W6.32 (IgG2a anti-HLA I), 5B14 (IgM anti-B7H3), L14 (IgG2a anti-nectin 2). The following purchased antibodies were used in this study: anti-CD56/NCAM-1 (IgG1), anti-CD3 (rabbit IgG) (Thermo Scientific), anti-ESA (IgG1), anti-Ca125 (IgG1, anti-MUC16) (Novocastra Laboratories Ltd.), anti-CD90 (IgG1, BD-Pharmingen), and mixture of FITC-labeled CD3 plus PC5-labeled CD56, FITC-labeled CD14 and FITC-labeled CD20 (Beckman-Coulter, Immunotech).

\section{NK Cell Isolation from $P B$ and $P F$}

PB and PF were collected at the time of surgery. Informed consent was provided according to the Declaration of Helsinki. Mononuclear cells from heparinized PB or PF were obtained by density-gradient centrifugation using Ficoll (Sigma, St. Louis, Mo., USA) [15]. NK cell analysis by flow cytometry was made on gated CD56 ${ }^{+} / \mathrm{CD}^{-}$cells.

\section{Tumor Cell Isolation from PF or a Tumor Solid Mass and Human Neuroblastoma Cell \\ Lines}

Tumor cells from PF were obtained by density-gradient centrifugation over Ficoll. Solid neuroblastoma tissue was minced into $1-\mathrm{cm}^{3}$ fragments that were then disgregated with sterile tweezers. We used human neuroblastoma cell lines HTLA-230 and SK-N-BE in comparison with PF and tumor mass neuroblastoma cells [13]. Cell lines were kept at low passage, returning frequently to original frozen stocks.

\section{Immunohistochemical Staining and Cytofluorimetric Analysis}

Histological samples, obtained from resected NB tumor mass, were evaluated by immunohistochemistry on frozen sections and paraffin sections. The sections were incubated with primary antibodies at room temperature for $2 \mathrm{~h}$ and with labelled polymer-HRP antimouse for 30 min (EnVision plus; Dako). The sections were incubated with DAB (Dako) and counterstained with hematoxylin (Carlo Erba). Cells acquisition was performed on a FACSCalibur flow cytometer (Becton-Dickinson, Mountain View, Calif., USA) and data analyzed using the Cell Quest software (Becton-Dickinson).

\section{Cytolytic Activity}

PBMCs (exposed or not to rIL-2) were tested for cytolytic activity against NK-susceptible tumor target cells K562 (human erythroleukemia cell line) in a 4-hour ${ }^{51} \mathrm{Cr}$-release assay as previously described.

\section{Results}

NK Cell Analysis

First, we analyzed the surface phenotype of NK cells isolated from PF as compared to autologous PB-NK cells and healthy controls. Importantly, we observed a substantial increase of CD56 bright NK cells in PF. In line with this result, the CD16 receptor (primarily expressed by CD56dull NK cells), was significantly reduced on PF-NK cells (fig. 1a, A-C). Moreover, PF-NK cells displayed an increase in the expression of the activation marker CD69 
Tabellini et al.: Primitive Neuroectodermal Tumor in an Ovarian Cystic Teratoma: Natural Killer and Neuroblastoma Cell Analysis

(75 vs. $25 \%$ in PB), and a reduction of CXCR1 chemokine receptor (27 vs. $82 \%$ in PB) (data not shown) [12].

Next, we analyzed the expression of the major triggering NK receptors, including NKp46, NKp30, DNAM-1 and NKG2D (fig. 1a, A-C). We observed that the mean fluorescence intensity (MFI) of NKp46 (but not of NKp30 and NKG2D) was higher on NK cells derived from PF as compared to PB (MFI values: 329 vs. 173) (fig. 1a, B, C). Remarkably, both the MFI and the percent of DNAM-1+ NK cells were substantially decreased in PF as compared to $\mathrm{PB}$ and healthy donors (fig. 1a, C).

The limited number of tumor cells isolated from PF did not allow us to perform cytotoxicity assays between PB and PF-NK cells and autologous tumor cells. However, cytotoxicity experiments were conducted using PB- and PF-NK cells against K562, a tumor target known to be recognized via distinct sets of triggering NK receptors, including NKp30 and DNAM-1. These functional assays demonstrated an impaired cytotoxic activity of PF-NK cells when compared with NK cells derived from the patient and from healthy donors (fig. 1b).

\section{Analysis of Neuroblastoma Cells}

Immunohistochemical analysis of the tumor mass revealed that NB cells expressed CD56 (fig. 2b) and B7-H3 (fig. 2a), a glycoprotein proven to be useful to identify NB cells [13]. The tumor mass did not express the Ca125 molecule, a common marker of epithelial ovarian cancer cells (fig. 2c), although a soluble form of this marker was present in the patient's serum.

Previous studies indicated that the DNAM-1 receptor plays a crucial role in the NKmediated recognition of NB cells [9]. Thus, we analyzed the expression of the DNAM-1 ligands (PVR and nectin-2) on tumor cells. In histological sections, PVR and nectin-2, although detected on endothelial cells of intratumoral neoplastic vessels, were totally absent on NB cells (fig. 2d, e). In line with these results, cytofluorimetric analysis of tumor cells freshly isolated from PF, indicated that NB cells did not express PVR and displayed lowsurface positivity for nectin-2 (table 1).

In table 1, the cytofluorimetric data obtained with a panel of different mAbs on freshly and primary cultured NB cells derived both PF and solid tumor are summarized. For comparison data obtained with two human NB cell lines (HTLA-230 and SK-N-BE) are reported. It can be seen that the DNAM-1 ligands PVR and nectin- 2 were negative/weakly expressed on freshly isolated NB cells, while they were highly positive on the two NKsusceptible NB cell lines (HTLA-230 and SK-N-BE). Remarkably, freshly isolated and primary cultured NB cells derived from PF expressed high levels of HLA class-I molecules (that were absent and weakly positive in HTLA-230 and SK-N-BE NB cell lines, respectively). Finally, both freshly isolated and primary cultured NB cells, as well as the two NB cell lines, were positive for CD56, CD90 and B7-H3.

\section{Discussion}

Altogether, our data show that the tumor carried by this patient displays a surface phenotype suggestive of a high resistance to NK-mediated lysis. This conclusion could be drawn based on the finding that NB cells express different ligands for inhibitory NK receptors including HLA class-I and B7-H3 [13]. Importantly, the tumor is also lacking crucial ligands for activating NK receptors such as PVR. Finally, the NK cells derived from the PF of the patient display lower expression of some important activating receptors including DNAM-1 and CD16 as well as a reduced cytolytic activity. 
Tabellini et al.: Primitive Neuroectodermal Tumor in an Ovarian Cystic Teratoma: Natural Killer and Neuroblastoma Cell Analysis

The altered expression of the DNAM-1 receptor observed in our study is reminiscent of that reported by Carlsten et al. [14], who showed DNAM-1 downmodulation on PF-derived NK cells from ovarian carcinoma patients. However, at present, it is difficult to establish if the loss of DNAM-1 expression detected in our case was caused by exposure to ligands. In this regard, the analysis of DNAM-1 ligands on NB cells derived from the tumor mass and from PF did not show a substantial expression of PVR on NB cells; we only detected a weak positivity for nectin-2. This ligand, at variance with PVR, may not be relevant in determining the magnitude of NK cell-mediated killing of neuroblasts [9] but it might be involved in the downregulation of DNAM-1 on NK cells.

PF-NK cells displayed additional modifications in the expression of other important surface NK cell molecules, such as a reduction of CD16, an increase of CD69 expression and an altered proportion between CD56 bright and CD56 dull $\mathrm{NK}$ cell subsets resulting in an increase in the percentage of CD56 bright $\mathrm{NK}$ cells (expressing high levels of NKp46) as compared to freshly isolated PB-NK cells. A similar alteration of the proportion between CD56 $6^{\text {bright }}$ and CD56 dull subsets was reported in ovarian carcinoma patients by Carlsten et al. [14].

Regarding the expression of ligands for inhibitory NK receptors, we show that the tumor of this patient expressed substantial amounts of HLA class-I molecules. Remarkably, previous studies demonstrated that freshly isolated neuroblasts from childhood patients either lacked or expressed negligible amounts of these molecules [9]. It is well established that the defective expression of HLA class-I molecules represents a favorable condition for allowing NK-mediated lysis, because NK cells express potent inhibitory receptors specific for these molecules such as KIR and CD94/NKG2A [8]. Thus, the tumor of our patient may escape the NK-mediated control because of its HLA class- $\mathrm{I}^{+}$phenotype.

Importantly, these NB cells also expressed B7-H3, a surface glycoprotein belonging to the B7 family that was shown to inhibit the NK-mediated lysis of NB by interacting with a still undefined inhibitory receptor expressed by NK cells [13]. Thus, the expression of B7-H3 on these NB cells may represent a further mechanism to escape the NK-mediated control [13].

The mechanisms that control susceptibility of given target cells to NK cell-mediated lysis are not only based on inhibitory interactions, but involve also the interaction of triggering NK receptors with their ligands. As mentioned above regarding the expression of DNAM-1 ligands, NB cells from our patient lack PVR. The absence of this molecule was found to be associated with poor susceptibility of neuroblasts to NK-mediated lysis [9]. Thus, also in this case, NB cells from our patient appear to display phenotypic features that are protective against NK cells.

In conclusion, our data provide the first characterization of NK cells and autologous tumor cells in a rare case of neuroblastoma arising in an ovarian cystic teratoma from an adult patient. While in childhood, approximately $50 \%$ of the NB appeared to be susceptible to NK-mediated lysis and thus eligible for a possible NK-based immunotherapy, in the present case the activity of NK cells against the tumor appears to be hampered by the phenotypic characteristics of both NB and NK cells.

\section{Acknowledgments}

The histological diagnosis of the neuroblastoma case by the $1^{\circ}$ Servizio di Anatomia Patologica, Spedali Civili, Brescia, Italy, is gratefully acknowledged. This work was supported by grants awarded by the Ministero dell'Istruzione dell'Università e della Ricerca (PRIN 
Tabellini et al.: Primitive Neuroectodermal Tumor in an Ovarian Cystic Teratoma: Natural Killer and Neuroblastoma Cell Analysis

2008 PTB3HC_002), by the Centro di Studio e Ricerca 'Quality and Technology Assessment, Governance and Communication Strategies in Health Systems', by the Associazione Italiana Ricerca sul Cancro, IG project No. 10643 (A.M.), by the Special Project 5x1000 No. 9962 (A.M.) and by the Progetto Fondazione Carige, prat. No. 2013.0275-11 (E.M.).

\section{Disclosure Statement}

A. Moretta is a founder and shareholder of Innate-Pharma (Marseille, France). The remaining authors have no competing financial interests to declare.

\section{References}

1 Kleinman GM, Young RH, Scully RE: Primary neuroectodermal tumors of the ovary. A report of 25 cases. Am J Surg Pathol 1993;17:764-778.

2 Morovic A, Damjanov I: Neuroectodermal ovarian tumors: a brief overview. Histol Histopathol 2008;23:765-771.

-3 Reid HA, van der Walt JD, Fox H: Neuroblastoma arising in a mature cystic teratoma of the ovary. J Clin Pathol 1983;36:68-73.

4 Muhlstein J, Rodriguez-Dahlhoff S, Marie B, Fouyssac F: Primary ovarian neuroblastoma. J Pediatr Adolesc Gynecol 2010;23:263-266.

5 Vivier E, Raulet DH, Moretta A, Caligiuri MA, Zitvogel L, Lanier LL, Yokoyama WM, Ugolini S: Innate or adaptive immunity? The example of natural killer cells. Science 2011;331:44-49.

6 Lanier LL: Up on the tightrope: natural killer cell activation and inhibition. Nat Immunol 2008;9:495-502.

7 Moretta A, Bottino C, Vitale M, Pende D, Cantoni C, Mingari MC, Biassoni R, Moretta L: Activating receptors and coreceptors involved in human natural killer cell-mediated cytolysis. Annu Rev Immunol 2001;19:197223.

8 Moretta A, Bottino C, Vitale M, Pende D, Biassoni R, Mingari MC, Moretta L: Receptors for HLA class-I molecules in human natural killer cells. Annu Rev Immunol 1996;14:619-648.

-9 Castriconi R, Dondero A, Corrias MV, Lanino E, Pende D, Moretta L, Bottino C, Moretta A: Natural killer cellmediated killing of freshly isolated neuroblastoma cells: critical role of DNAX accessory molecule-1poliovirus receptor interaction. Cancer Res 2004;64:9180-9184.

-10 Sivori S, Parolini S, Marcenaro E, Castriconi R, Pende D, Millo R, Moretta A: Involvement of natural cytotoxicity receptors in human natural killer cell-mediated lysis of neuroblastoma and glioblastoma cell lines. J Neuroimmunol 2000;107:220-225.

11 Singh M, Arora P, Singh S, Bohara S, Khurana N, Zutshi V, Kumar R: Adult neuroblastoma of the ovary: a rare tumor with review of literature. J Cancer Res Ther 2010;6:367-369.

12 Parolini S, Santoro A, Marcenaro E, Luini W, Massardi L, Facchetti F, Communi D, Parmentier M, Majorana A, Sironi M, Tabellini G, Moretta A, Sozzani S: The role of chemerin in the colocalization of NK and dendritic cell subsets into inflamed tissues. Blood 2007;109:3625-3632.

-13 Castriconi R, Dondero A, Augugliaro R, Cantoni C, Carnemolla B, Sementa AR, Negri F, Conte R, Corrias MV, Moretta L, Moretta A, Bottino C: Identification of 4Ig-B7-hH as a neuroblastoma-associated molecule that exerts a protective role from an NK cell-mediated lysis. Proc Natl Acad Sci USA 2004;101:12640-12645.

14 Carlsten M, Norell H, Bryceson YT, Poschke I, Schedvins K, Ljunggren HG, Kiessling R, Malmberg KJ: Primary human tumor cells expressing CD155 impair tumor targeting by down-regulating DNAM-1 on NK cells. J Immunol 2009;183:4921-4930.

15 Marcenaro E, Cantoni C, Pesce S, Prato C, Pende D, Agaugué S, Moretta L, Moretta A: Uptake of CCR7 and acquisition of migratory properties by human KIR+ NK cells interacting with monocyte-derived DC or EBV cell lines: regulation by KIR/HLA-class I interaction. Blood 2009;114:4108-4116. 
Tabellini et al.: Primitive Neuroectodermal Tumor in an Ovarian Cystic Teratoma: Natural Killer and Neuroblastoma Cell Analysis

Table 1. Flow cytometric analysis of freshly isolated and primary cultured neuroblastoma cells derived from PF and solid tumor

\begin{tabular}{llllll}
\hline & FNBC PF & PCNB PF & PCNB T & SK-N-BE & HTLA-230 \\
\hline CD90 & +++ & +++ & +++ & +++ & +++ \\
ESA & ND & - & - & - & - \\
CD56 & + & + & $+/-$ & +++ & +++ \\
HLA-I & +++ & +++ & +++ & + & - \\
B7/H3 & $+/-$ & + & $+/-$ & ++ & +++ \\
PVR & - & ND & ND & +++ & +++ \\
Nectin-2 & $+/-$ & ND & ND & ++ & +++ \\
\hline
\end{tabular}

$-=0-10 \%$ positive tumor cells; $+/-=10-25 \%$ positive tumor cells; $+=$ $26-50 \%$ positive tumor cells; $++=51-75 \%$ positive tumor cells; $+++=$ $76-100 \%$ positive tumor cells.

$\mathrm{ND}=$ Not determined; FNBC PF = neuroblastoma cells freshly derived from PF; PCNB PF = primary cultured neuroblastoma cells derived from PF; PCNB T = primary cultured neuroblastoma derived from solid tumor; SK-N-BE and HTLA-230 = human neuroblastoma cell lines. The analysis of FNBC PF, PCNB PF and PCNB T was performed on a CD90+-gated cell population. 
Tabellini et al.: Primitive Neuroectodermal Tumor in an Ovarian Cystic Teratoma: Natural Killer and Neuroblastoma Cell Analysis

a Phenotypic analysis of NK cells

A

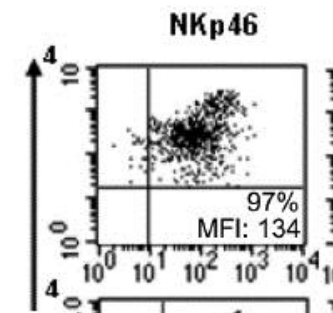

NKp30

NKG2D

DNAM-1

CD 16

B
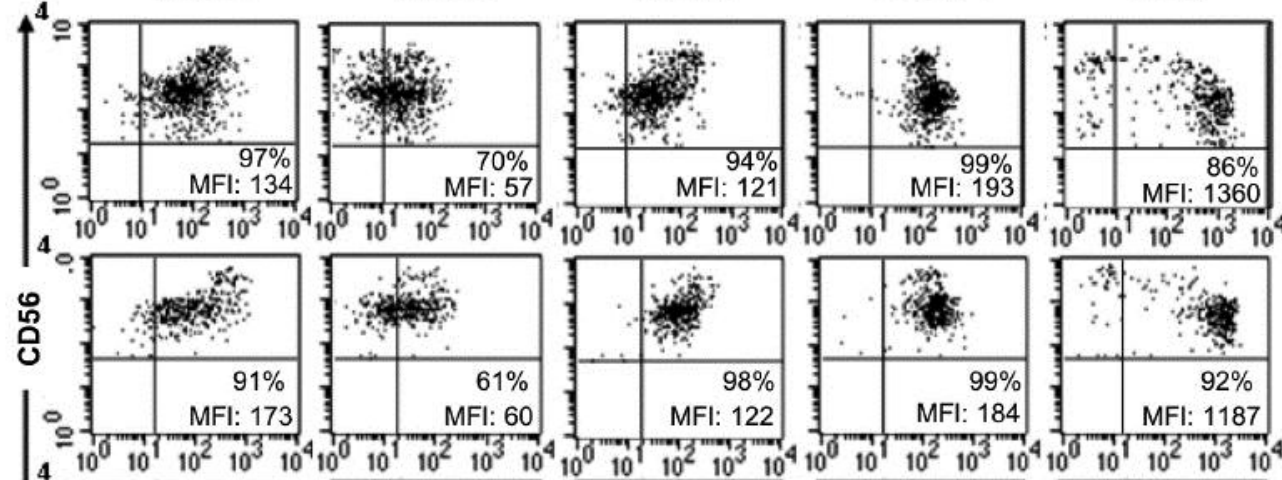

C
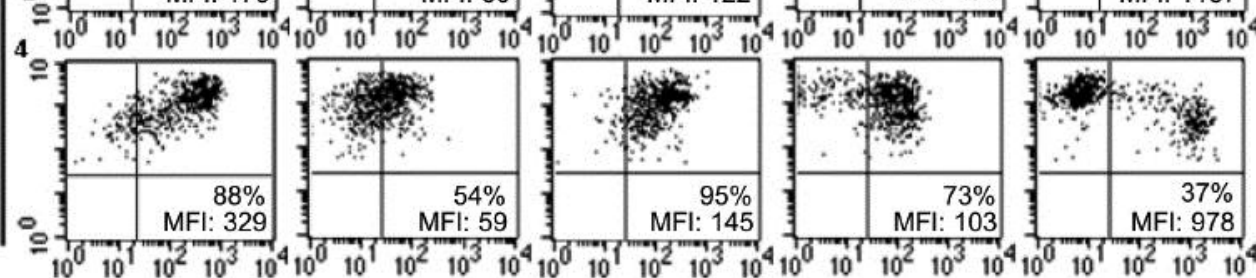

\section{b Cytotoxic activity of NK cells}
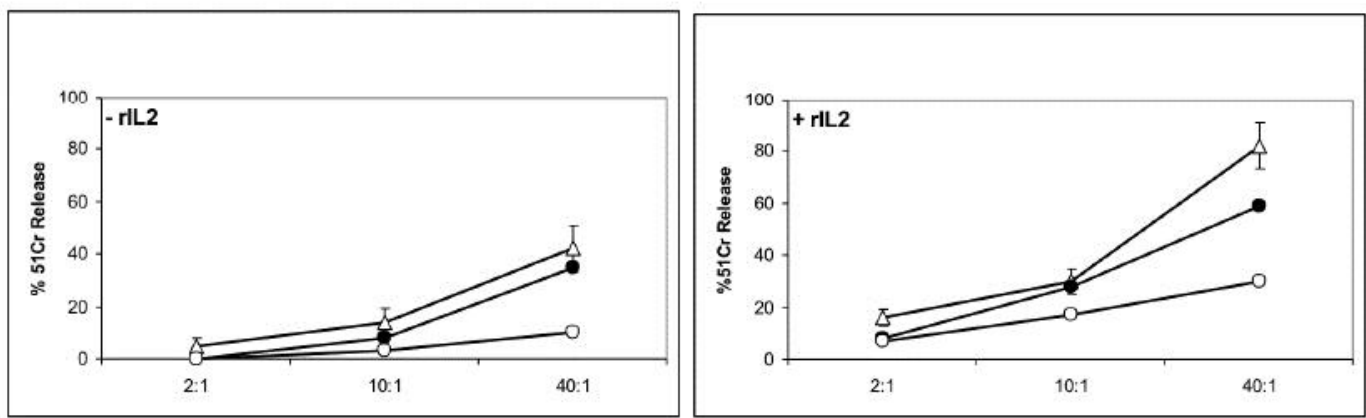

Fig. 1. a Phenotypic analysis of PF-NK cells. Expression of NK cell receptors gated on CD56+CD3-CD14CD20- cells from $\mathrm{PB}$ of a representative healthy donor (A) of 6 distinct subjects examined and from PB (B) and $P F(C)$ of the patient. Numbers indicate the percentage and MFI of receptor-positive cells. $\mathbf{b}$ Cytotoxic activity of NK cells against the K562 target cell line. Freshly isolated mononuclear PB and PF cells derived from the patient (black and white circles, respectively) and from healthy donors (triangles) were comparatively analyzed against the K562 target cell line either without (left) or with (right) overnight incubation in the presence of rIL-2 at different E/T ratios. Data about healthy donors indicate the mean \pm SD of 6 different control subjects. 


\section{Case Reports in Oncology}

\begin{tabular}{l|l}
\hline Case Rep Oncol 2014;7:70-78 & \multicolumn{2}{l}{$\begin{array}{l}\text { C 2014 S. Karger AG, Basel } \\
\text { www.karger.com/cro }\end{array}$} \\
\hline DOI: 10.1159/000357802 &
\end{tabular}

Tabellini et al.: Primitive Neuroectodermal Tumor in an Ovarian Cystic Teratoma: Natural Killer and Neuroblastoma Cell Analysis
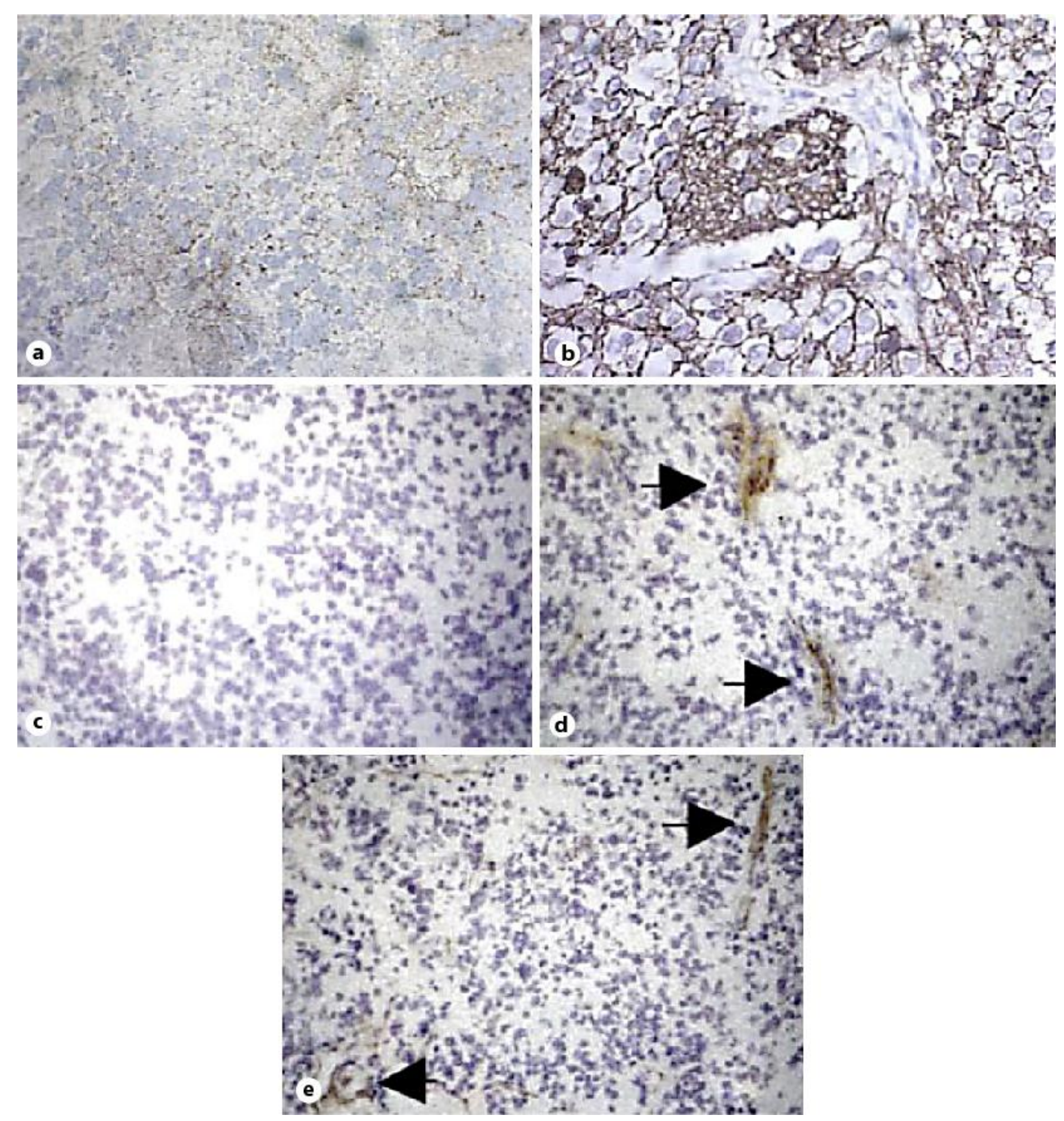

Fig. 2. Immunohistochemical analysis of a primitive neuroblastoma. Staining was performed with antibodies anti-B7-H3 (a), anti-CD56 (b), anti-Ca125 (c), anti-PVR (d) and anti-nectin (e). In d and e, positive staining is detected in the intratumoral vessels (arrowheads). Immunostaining was performed on paraffin-embedded tissue sections for CD56, Ca125, PVR and nectin and on frozen sections for B7-H3. Nuclei were counterstained with hematoxylin (blue). a, b, 40× original magnification, c-e $20 \times$ original magnification. 